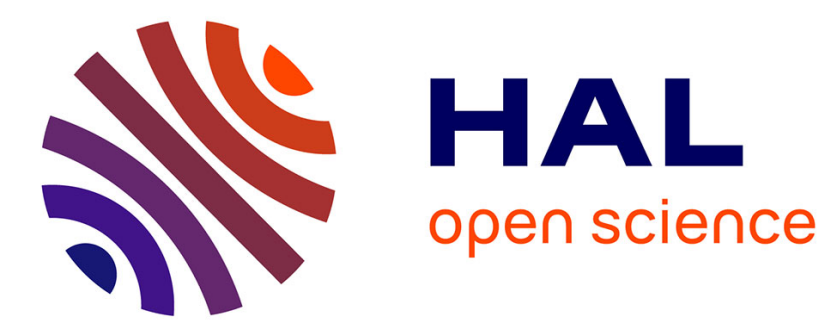

\title{
Sans pathos. Des Kongo Astronauts, de Lamyne M.
} Dominique Malaquais

\section{To cite this version:}

Dominique Malaquais. Sans pathos. Des Kongo Astronauts, de Lamyne M.. Critique, 2020. halshs03100460

\section{HAL Id: halshs-03100460 \\ https://shs.hal.science/halshs-03100460}

Submitted on 6 Jan 2021

HAL is a multi-disciplinary open access archive for the deposit and dissemination of scientific research documents, whether they are published or not. The documents may come from teaching and research institutions in France or abroad, or from public or private research centers.
L'archive ouverte pluridisciplinaire HAL, est destinée au dépôt et à la diffusion de documents scientifiques de niveau recherche, publiés ou non, émanant des établissements d'enseignement et de recherche français ou étrangers, des laboratoires publics ou privés. 


\section{Sans pathos \\ Des Kongo Astronauts, de Lamyne $\mathrm{M}$}

Partout en Europe s'immisce le populisme. Ce populisme et l'électorat qu'il courtise présentent l'immigration en provenance de l'Afrique comme un danger criant ${ }^{1}$. Plongeant sa source dans un racisme systémique, la désignation de cette cible repose sur trois prémisses fausses: qu'entre en Europe une véritable déferlante venue d'Afrique; que l'origine en est avant tout d'ordre économique et non politique; qu'être à l'abri de l'insécurité économique ne relève pas des droits humains. À gauche, ces affirmations sont rejetées en ce qu'elles sont constitutives du projet néolibéral. Au centre, on tend à s'écarter de considérations structurelles de cet ordre au profit d'arguments plus propres à attirer les suffrages du grand nombre; d'où des campagnes focalisées d'abord sur les risques mortels qu'encourent ceux qui cherchent à fuir le continent africain et sur la déshumanisation qui les attend. Dénoncer ces conditions est indispensable, mais se cantonner à cela peut être délétère : c'est risquer de faire des gens dont ces campagnes "prennent la défense» des figures de pathos. C'est les priver d'agentivité, leur refuser le statut de protagonistes en prise sur leur sort, tout catastrophique qu'il puisse s'annoncer.

En Afrique et dans les diasporas, cette problématique est prise à bras le corps par des artistes contemporains. Via des œuvres précisément axées sur le problème de l'agentivité, elles et ils rejettent la désautonomisation. Faisant fi d'un repli dans la tristesse ou la pitié, et se détournant du sensationnel, ces artistes déploient une critique acerbe de la violence néolibérale et du discours sur l'immigration qui en exsude. Je m'intéresserai ici à deux séries de travaux qui s'inscrivent

1. S. Smith, La Ruée vers l'Europe. La jeune Afrique en route pour le Vieux Continent, Paris, Grasset, 2018. 
en faux contre ce discours. Elles sont différentes sur bien des plans: par leurs matériaux, leurs formes, leurs contenus et leurs intentions et ne ressortissent pas à un genre, à un art de l'immigration - en fait, seule l'une des deux se rapporte directement à cette question. Ce qui les rapproche, c'est plutôt une vision de l'Afrique comme plateforme de mouvement - mouvement de personnes, d'objets et d'idées entre ce continent et le reste du monde, en opposition active aux structures politiques et économiques qui s'appliquent à le freiner.

\section{Après Schengen?}

En 2019, le collectif d'artistes Kongo Astronauts, basé à Kinshasa, produit une série de performances et de photos intitulées Après Schengen. Ce travail a pour toile de fond un Boeing désossé échoué aux abords de la capitale congolaise. Sur chacune des photos (il y en a onze) figure un astronaute portant combinaison, casque et bottes dorés. Il apparait successivement au centre d'une cabine vidée de tout élément, dans un train d'atterrissage, sur une aile, dans l'encadrement d'une issue ou s'éloignant de la carcasse de l'avion (Fig. 1). C'est le performeur Michel Ekeba, photographié par Eléonore Hellio. Ensemble, ils ont fondé le collectif en 2013.

La présence d'Ekeba dans cet environnement se prête à deux interprétations contradictoires. Depuis la France, dans le contexte médiatique que l'on sait, on est à première vue tenté d'y voir une allégorie de l'immigration clandestine. Un mien collègue y a ainsi perçu l'expression d'une fuite désespérée. Depuis le Congo, la série suscite une tout autre lecture. Une année durant (2018-2019), à la suite d'une friction diplomatique entre la RDC et l'Union européenne, la Maison Schengen, centre de tri des demandes de visa pour l'Europe géré par la Belgique, a fermé ses portes. Déjà extrêmement difficile, l'obtention de visas devenait cauchemardesque. Dans ce cadre, le titre Après Schengen prend l'allure d'une question: et maintenant, où? Les photos répondent: vers les étoiles.

Si l'ambiguïté est intentionnelle - j'y reviendrai plus loin - il est clair que, dans le contexte plus large du travail des Kongo Astronauts, c'est la seconde lecture qui s'impose. 


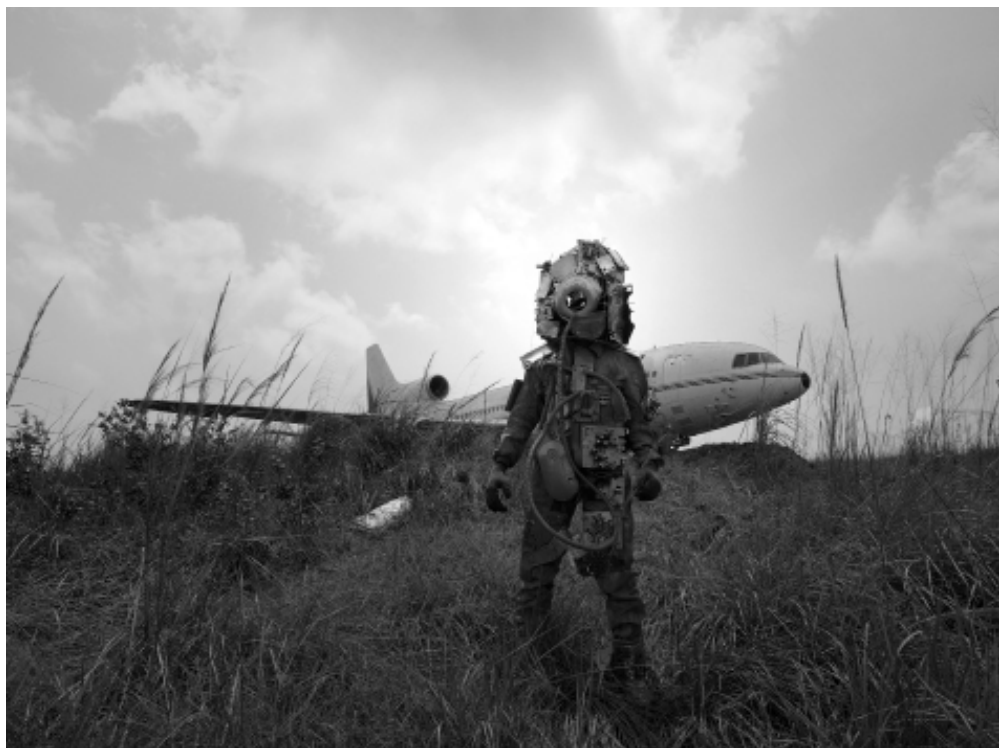

Fig. 1. Kongo Astronauts.

Sans titre - Série Après Schengen (2019).

Tirage argentique. Photo (C) Kongo Astronauts. 
Là, cependant, la voûte céleste est moins un lieu physique où les artistes aimeraient se rendre, qu'une arène métaphorique. Elle sert de plateforme pour aborder le problème de la décimation de la Terre par un modèle économique ancré dans le projet colonial et que le néolibéralisme va amplifiant. C'est ce que suggère l'habit d'Ekeba. De la tête aux pieds, sa tenue est constellée de pièces d'ordinateurs et de smartphones collectées sur les marchés et dans des décharges. Ces e-déchets, importés (souvent illégalement) du Nord et, de plus en plus, de l'Est, sont le produit de pratiques qui font du continent un dépotoir du numérique. Les matériaux choisis font aussi référence au pillage des ressources du Congo par les multinationales; les cartes-mères, câbles et batteries qui recouvrent le vêtement de l'astronaute contiennent des métaux précieux - cuivre et or, zinc, tantale, lithium - extraits en RDC au prix de violences inqualifiables par des conglomérats étrangers travaillant main dans la main avec une élite locale corrompue.

Dans certaines parties du Congo, le dumping et le pillage qu'évoquent les performances d'Ekeba et les photos de Hellio créent une situation quasi apocalyptique. Les Kongo Astronauts dénoncent cela. Toutefois, plutôt que de mettre en exergue cette situation, le collectif en fait ressortir les causes. Cette approche est caractéristique de l'ensemble de son travail. Bien que souvent détonnant, en écho à la brutalité du capitalisme extractif, celui-ci évite le pathos et tombe rarement dans le voyeurisme. En cela, il se démarque de réalisations centrées sur le Congo qui font appel, précisément, à ce registre. Une comparaison d'Après Schengen avec des performances mises en scène pour Système $K$ (2018), film du réalisateur Renaud Barret consacré à l'art contemporain à Kinshasa ${ }^{2}$, et avec un travail photographique de Richard Mosse sur le $\mathrm{Congo}^{3}$ est à cet égard éclairante. Certains des travaux présentés dans Système $K$ visent explicitement à choquer. Exemple: une performance de Yannos Majestikos (Yannick Makanka Tungaditu), dans laquelle l'artiste, plongé dans un bain de sang coagulé, est véhiculé par les rues de Kinshasa. La série Infra de Mosse

2. Voir dans ce même numéro l'article de Julie Peghini.

3. R. Mosse, Infra, New York, Aperture Foundation, 2011. 
(2011), photographiée dans un Congo oriental en pleine guerre, joue également sur la valeur choc. Des paysages verdoyants y sont montrés comme saturés de sang par le truchement d'une pellicule infrarouge qui remplace les verts par des roses criards.

Le choix que font les Kongo Astronauts de tenir à distance l'émotion sur laquelle s'appuient nombre d'images représentant le Congo s'applique également au regard qu'ils portent sur l'immigration. Si, face à une Maison Schengen verrouillée, leur réponse est de viser les étoiles, ils semblent aussi poser que l'Europe n'a plus guère de pertinence, que ce soit comme lieu où vivre (du moins sur le long terme) ou comme espace à partir duquel penser le monde. C'est là un départ pour Hellio dans ses travaux sur le Congo. Mowoso, collectif (aujourd'hui dissous) qu'elle avait fondé en 2007 avec le vidéaste Dicoco Boketshu, s'intéressait tout particulièrement à la thématique de l'exil vers l'Europe ${ }^{4}$. On ne veut pas dire par-là que l'exil n'est pas un sujet capital pour les Kongo Astronauts - à preuve leur site Internet ${ }^{5}$ - mais que l'exil, pour eux, tient à bien plus qu'au lieu physique. C'est avant tout un lieu de «résistance aux ghettos psychiques [nés] des réalités postcoloniales» (Hellio) ${ }^{6}$. Partir par l'esprit est tout aussi pertinent que le faire par le corps. Michel Ekeba le souligne pour sa part. Il qualifie sa transformation en astronaute d'éprouvante et dit qu'elle modifie son état de conscience. Le poids de la tenue et la chaleur qu'elle dégage dans le climat équatorial de Kinshasa suscitent en lui un état de transe qui altère radicalement sa perception et il se transforme ainsi en un être venu d'une autre planète, dont les yeux voient ce qui échappe au commun des mortels ${ }^{7}$.

4. D. Malaquais, «On the urban condition at the edge of the $21^{\text {st }}$ century: Time and space in question», Social Dynamics, 2018, vol. 44, $\mathrm{n}^{\circ} 3$, p. $425-437$.

5. Le collectif est, à cet égard, explicite: «[L]es apparitions cosmiques et les fictions polysémiques de Kongo Astronauts nous engagent à porter un regard multidimensionnel sur différentes formes d'exil et tactiques de survie.» Voir en ligne: https://kongoastronauts.wordpress.com/aboutkongo-astronauts-kinshasa-rdcongo-2013-2019/.

6. Ibid.

7. D. Malaquais, «Kongo Astronauts : collectif embarqué», Multitudes, hiver 2019, n $^{\circ} 77$, p. 20-26; ici, p. 22. 
On reconnaît là l'influence clé, revendiquée comme telle par les Kongo Astronauts, de l'artiste visionnaire Sun Ra - un «ancêtre», dit Hellio. Si celle-ci, d'emblée, a initié Ekeba à l'esthétique et à la philosophie de l'Arkestra (l'ensemble fondé par Sun Ra au milieu des années 1950) et, plus largement, à l'afrofutursime, avec Ekeba, elle-même a découvert l'histoire de l'exploration spatiale au Congo - et en particulier les ambitions de Mobutu en la matière. Ensemble, au Congo et ailleurs, ils collaborent avec des artistes et des penseurs pour qui l'espace est le lieu de tous les imaginaires. Ainsi, à Kinshasa, ils travaillent avec Bienvenu Nanga et Danniel Toya, artistes qui appartiennent à deux générations différentes, mais qui, tous deux, fabriquent de complexes robots/ cyborgs à taille humaine; avec la musicienne Céline Banza qui, dans Prédic(a)tion, film récent des Kongo Astronauts, incarne le rôle d'une cosmonaute perdue dans l'espace; et avec le musicien/compositeur/poète/inventeur Bebson Elemba (dit Bebson de la Rue), dont les «cabines de télétransport» et les «environnements interstellaires ${ }^{8}$ font souvent irruption dans les productions du collectif. En dehors de la République Démocratique du Congo, le collectif est en fréquent dialogue avec des acteurs culturels qui voient dans l'espace l'au-delà d'une humanité abîmée ${ }^{9}$.

Si Éléonore Hellio s'emploie à traduire l'expérience extracorporelle de Michel Ekeba, c'est qu'elle entrevoit là une manière alternative d'aborder un monde déglingué: elle veut que nous le voyions par les yeux de l'astronaute. Abordées sous cet angle, les photos de la série After Schengen ne constituent pas, comme le pensait mon collègue, une image de l'exil - exil désiré, tenté, désespéré - mais une image depuis l'exil : un exil déjà entamé par la pensée, loin de la violence d'un siècle et demi d'extractivisme sauvage; bien loin aussi des représentations spectaculaires et pathétiques du chaos produites pour une large part à l'intention de la société de consommation.

8. Ibid., p. 20.

9. Ainsi de l'équipe de la Pan-African Space Station (PASS), emmenée par Ntone Edjabe; de MIDBO, un festival cinématographique de Bogotá dont l'édition 2018 s'est tenue en partie dans un télescope géant; ou encore de l'Observatoire de l'espace (Centre national d'études spatiales, Paris). 
Des photos de la série Après Schengen se dégage un très net sentiment d'absurdité. Elles ont été prises sur une colline surplombant une réserve naturelle de dix mille hectares, le Parc de la vallée de la N'sele, créé en 2018 par le gouvernement du président d'alors, Joseph Kabila. Peuplé d'animaux sauvages importés, ce parc se présente (en ligne) comme un espace où le visiteur peut communier avec «des animaux qui représentent cette partie du monde dans notre imaginaire à tous et qui étaient pourtant bel et bien disparus ${ }^{10}$ » et comme un lieu idéal pour organiser des retraites de team-building à l'intention d'équipes commerciales ${ }^{11}$. L'avion échoué, s'avèret-il, n'est pas du tout une épave abandonnée, mais un appareil mis à la retraite et hissé pièce par pièce sur la hauteur. Tout cela à l'initiative de la première dame, Olive Lembe di Sita, dont l'objectif était de transformer le jet en restaurant de luxe :

À l'intérieur de cet avion [...] les visiteurs, coupés du monde, pourront voyager à leur manière sous l'osmose d'un déjeuner 5 étoiles en première classe dans un vol le moins fallacieux qui puisse exister. [...] À côté sera érigée une tour de contrôle fictive avec chambre à coucher VIP, une terrasse panoramique avec baie vitrée offrant une vue splendide sur le paysage ${ }^{12}$.

Le choix de ce décor comme arrière-plan de la série After Schengen est brillant. Ce qui, vu hors contexte, se lit comme un espace de désespoir - un avion sur lequel chercherait à s'embarquer un passager clandestin - est en fait «une première classe» en devenir. Lastronaute a atterri dans un pseudo-Congo disneyesque, inventé à l'usage de touristes étrangers et d'une infime bourgeoisie locale. Le montre bien une publicité sur le site Congo Autrement où l'on voit des images du parc côte à côte avec un spot pour l'hôtel le plus huppé de Kinshasa, le Fleuve Congo, accompagné du logo de Congo Airways et de son slogan, «le plaisir de voyager ${ }^{13} »$. On

10. Voir en ligne: https://www.congo-autrement.com/page/rdctourisme-2/tourisme-kinshasa-le-parc-de-la-vallee-de-la-n-sele.html.

11. Voir enligne: https://www.youtube.comwatch?v=B6ngARr2nCc.

12. Voir en ligne: https://www.congo-autrement.com/page/rdctourisme-2/tourisme-kinshasa-le-parc-de-la-vallee-de-la-n-sele.html.

13. Ibid. 
rappellera ici, ce qui ne fera que corser l'absurdité ambiante, que Mobutu, lui aussi, avait jeté son dévolu sur la vallée de la N'Sele pour y créer un parc animalier, à proximité duquel il avait fait bâtir un gigantesque palais de marbre et une fausse pagode chinoise. Le restaurant cinq étoiles de la première dame n'est pas né de rien...

After Schengen répond avec ambiguïté, humour, sarcasme, consternation et colère aux systèmes économiques et politiques qui rendent possibles de telles élucubrations. À cela s'ajoute un courant de tension sous-jacent qui complique encore le propos. Eléonore Hellio et Michel Ekeba, duo binational, ne vivent pas de la même manière la bunkerisation de l'Union européenne. Hellio, citoyenne française, peut s'octroyer le plaisir de voyager; il n'en va pas de même pour le Congolais Ekeba. Elle peut se permettre d'imaginer un after Schengen; lui n'a guère d'autre choix. La violence d'un monde néolibéral qui divise pour régner est tout à la fois un thème qu'explorent les performances et les photos du collectif et une condition intrinsèque de leur production.

\section{Charme(s)}

La seconde série d'œuvres dont il sera ici question traite spécifiquement du mouvement face à la bunkerisation du Nord. Même si elle n'a pas pour focale la seule Europe, elle s'en prend à Frontex ${ }^{14}$ en particulier. Schengen est dans son viseur. Intitulée Gris-gris/marabout 3.0, la série est due à l'installation artist et performeur camerounais/français Lamyne M. Elle est constituée de 111 pièces allant de 9 à $110 \mathrm{~cm}$. Toutes sont le produit de collaborations. Conçue pour assurer la protection de personnes en transit entre l'Afrique et le reste du monde, chaque pièce a été élaborée par un religieux (un marabout dit Lamyne $\mathrm{M}$, d'où le nom de la série) en présence de l'artiste et sous sa direction. Quand les gris-gris sont exposés, ce processus de collaboration est souligné par l'inclusion d'un cartel indiquant sa double origine.

Chaque gri-gri a son propre objectif, auquel son titre fait référence et qui est précisé par quelques mots d'explication.

14. Rappelons que Frontex est la structure européenne de contrôle des frontières terrestres et maritimes (NdR). 
Titres et explications tout ensemble représentent un inventaire d'aspirations, d'espoirs nourris par femmes et hommes en quête de vies loin de l'Afrique. Ces objectifs vont du général au très précis. Massassy, créé en 2014 au Maroc par l'artiste et un marabout du nom d'Alvatan, offre «une assurance tous risques». Si certains gris-gris concernent un lieu spécifique (un pays, une région), Massassy couvre «le monde entier». Il en va de même de M'bappélepé, dont l'action est néanmoins plus ciblée. Fait en 2018 au Libéria par Lamyne M et le marabout Fekomanan, il garantit le «succès à l'étranger en tant que footballeur». Son propriétaire peut s'attendre à rencontrer «100\% de réussite» et aspirer à «gagner le Ballon d'or». Ciblé lui aussi, Bang-bang (Lamyne M et le marabout Gekefu, Tchad, 2019), qui promet l'accès à la Légion étrangère. L'entrée dans l'Union européenne est facilitée par un gri-gri élaboré en 2019, en Libye, avec le marabout Alnuur. Certes, comme le mot d'explication l'indique, ce talisman a pour champ d'action l'Europe, mais son pouvoir de déflexion peut agir jusqu'à la frontière mexicaine et, à ce titre, il relève d'un sous-groupe des gris-gris qui concerne l'immigration vers les Amériques. Alpharay (Lamyne $\mathrm{M}$ et le marabout sénégalais Modusalmaky, 2017) permet d'atteindre «un haut niveau d'intégration aux États-Unis». Les difficultés que rencontrent ceux qui souhaitent s'installer aux États-Unis (voire simplement s'y rendre en visite) sont aplanies par Forlamy (Lamyne M et le marabout Kunfayakun, Gabon, 2018), source de protection collective contre Donald Trump. Cette même amulette «protège de Viktor Orbán et de tous les extrémistes». Ce que fait également Horban, aussi appelé «anti-facho», vis-à-vis du même Orbán et d'autres acteurs de l'extrême droite, dont Marine Le Pen et Matteo Salvini (Lamyne M et le marabout Oguun, République du Congo, 2018).

La famille des gris-gris vient aussi en aide aux gens qui craignent de tomber entre les mains d'autres types d' "extrémistes»; ainsi Papakrapackchak (collaboration avec le marabout Kan Kan Game, Kenya, 2014), qui soustrait son propriétaire à la «radicalisation». Les gris-gris prennent en compte les aspirations et des "primo-arrivants» et de ceux dont l'installation remonte à plusieurs générations. Pour les premiers en quête de réunification familiale en France ou en Belgique, Lamyne $\mathrm{M}$ a créé Ittoto avec le marabout Touré 
(Mali, 2019). Pour les seconds il a conçu Ewossu (avec le marabout congolais Kofu, 2015), lequel confère à son détenteur, dont la famille vit en Russie depuis trois générations, l'aptitude à déchiffrer l'invisible.

Il existe plusieurs gris-gris sensibles au wifi, qui peuvent être mis à jour ou rechargés à distance par leurs marabouts en réponse à de nouveaux besoins de leurs propriétaires. D'où 3.0: on est ici en pleine futurologie.

Les gris-gris sont faits d'une vaste gamme de matériaux, parmi lesquels : tissus et fils; boutons, coquillages, pièces de monnaie, cuirs et peaux (taureau et vache, buffle, mouton, chèvre, antilope, chameau, serpent, iguane); parties d'animaux (cornes d'antilopes et de gazelles, griffes de félins et de vautours, fémurs d'hyènes, têtes de crocodiles, crânes d'aigles, plumes et becs d'oiseaux, carapaces de tortues) et, à l'occasion, animaux entiers (notamment deux caméléons) ; objets variés (boussole, torche, fourchettes, fioles, tours Eiffel miniatures, une paire de tennis, un gyrophare) ; jouets (petits soldats, bras et jambes de poupées); clés USB permettant de charger le gri-gri sur ordinateur; encre de diverses sortes servant à tracer des signes ésotériques ou encore mélangées à du liquide pour imprégner le talisman... Pour chaque charme, l'artiste donne une liste complète d'éléments constitutifs. Dans plusieurs cas, cette liste porte la mention «intérieur secret».

Cette mention ne concerne pas seulement l'observateur, qui n'est pas mis dans le secret, mais aussi l'artiste: Lamyne M ne sait pas ce que renferme le charme en question. Seul le marabout concerné en a l'intelligence. C'est là une caractéristique fondamentale de la série Gris-gris. Pour ludique qu'elles soient à première vue (j'y reviendrai), les $111 \mathrm{amu-}$ lettes sont aussi - et peut-être surtout - des objets de pouvoir. Elles sont chargées, chargées d'énergie spirituelle. Lamyne M est explicite à ce sujet: ce sont des objets agissants.

Cela dit, la série ne ressortit pas au seul domaine du spirituel. Lesthétique est pour beaucoup dans l'efficacité des gris-gris. Pour Lamyne M cela ne fait aucun doute: on parle ici d'art. Il veut que ses talismans soient appréciés pour leur apparence et leur toucher - pour leur impact sur les sens autant que pour leur capacité à agir sur le destin et la trajectoire de qui les détient. Lartiste fait appel à l'affect autant 


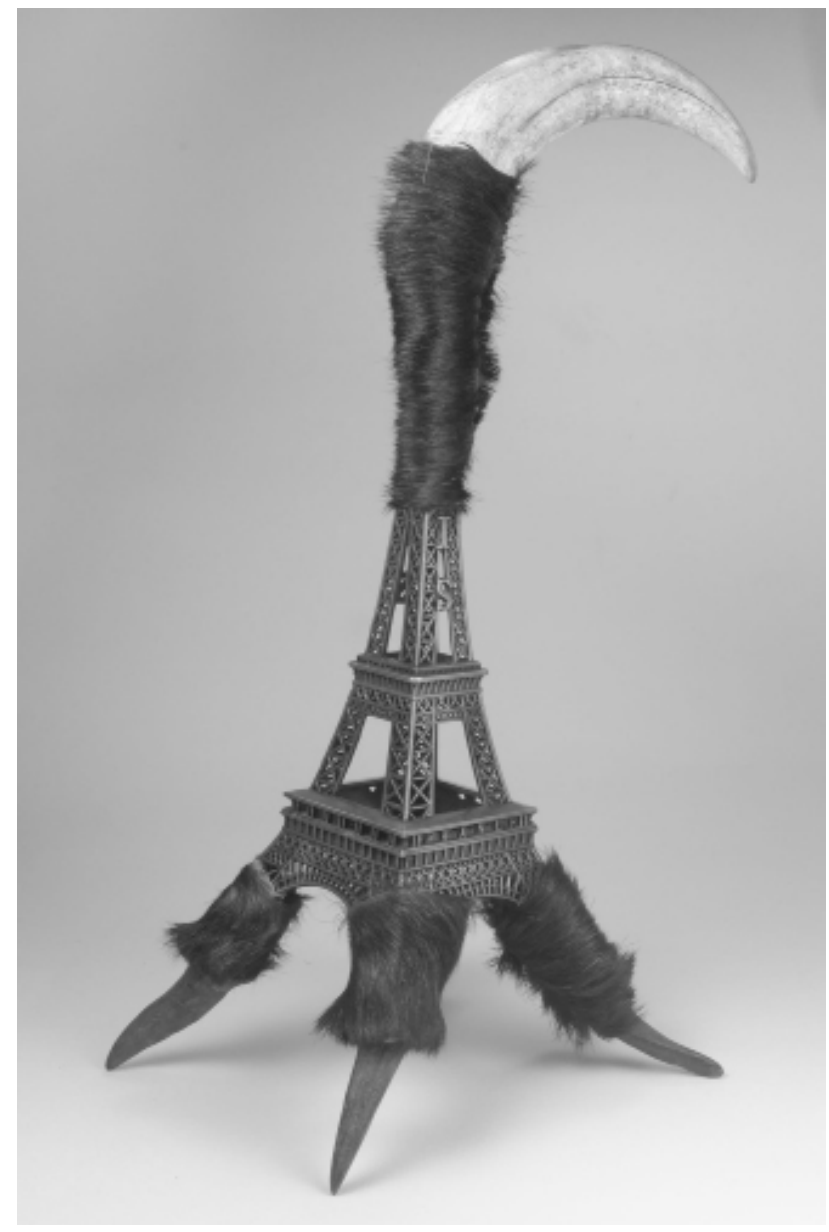

Fig. 2. Lamyne M.

Collaboration avec le marabout Touré, Mali.

Ittoto - (garantit la reunification familiale aux primoarrivants en France ou en Belgique). Série Grisgris/Marabout 3.0 (2017).

Reproduction en metal de la tour Eiffel, peau et corne de chèvre; intérieur secret. 32 × 20 x $15 \mathrm{~cm}$.

Collection privée. Photo (c) Lamyne M et Axis Gallery. 
qu'à l'effet. L'humour n'y est pas pour rien: une tour Eiffel miniature se dresse sur de petits pieds poilus (de minuscules cornes ourlées de fourrure) (Fig. 2); des baskets qui affichent une couronne de cornes de bouc tiennent à la fois de chaussures à crampon pour footballeur supersonique et de mini-fusées permettant d'escalader les gratte-ciel ${ }^{15}$; un ballot en peau d'iguane arborant des bras et des jambes de poupée évoque un superhéros mutant... Cet élément ludique s'articule à une critique mordante du Nord-forteresse qui se dégage des explications données par l'artiste. Il résulte de cela, du message politique qui sous-tend ces mêmes explications, de la charge spirituelle et esthétique des objets et de la fantaisie qui en émane une prise de position éloquente face à la violence du discours euro-américain sur la question de l'immigration.

Il convient de noter que nulle part Lamyne $M$ n'indique si les gris-gris sont à l'usage de voyageurs poussés par des considérations économiques ou d'individus ou de familles cherchant à fuir une tourmente politique. Nulle trace ici de la distinction entre l'économique et le politique qui fait le lit du discours sur la dite «crise migratoire», discours dans lequel le «migrant économique» est érigé en épouvantail. La série Gris-gris repose sur la prémisse que le mouvement entre les continents, quelles qu'en soient les motivations, est ancré dans un droit inaliénable. Les talismans de Lamyne M sont tout à la fois l'incarnation de cette vérité et un instrument

15. Si un second volume venait à être édité du remarquable ouvrage de Reynaldo Anderson et John Jennings, Cosmic Underground Grimoire of Black Speculative Discontent (San Francisco, Cedar Grove Publishing, 2018), le gri-gri en question y trouverait toute sa place. Lamyne $M$ ne se dit pas artiste afrofuturiste, mais certains concepts qu'il explore ont indubitablement des points d'intersection avec les discours de l'afrofuturisme. À preuve, notamment, l'incorporation de clés USB dans plusieurs talismans qui, on l'a vu, peuvent ainsi être branchés sur la toile et s'en trouver réactivés. Ces amulettes permettent non seulement de surmonter des obstacles physiques, mais aussi de franchir la fracture numérique. C'est là un sujet qu'explorent bien des auteurs d'œuvres littéraires afrofuturistes (dont le Camerounais Lionel Manga, dans un récit intitulé «Are you experienced?» qu'il a récemment publié en hommage aux Kongo Astronauts : L. Manga, «Are you experienced? », Multitudes, $\mathrm{n}^{\circ} 77$, p. 33-35). 
d'agentivité, déployé pour et par ceux qui entendent faire leur une liberté qu'on leur refuse.

Ni comme émotion, ni comme méthode de persuasion, le pathos n'est présent dans les travaux dont il a été question ici. Il est écarté dans After Schengen et Gris-gris pour mettre en relief la volonté et la capacité d'agir de l'individu. Ce qui ne veut pas dire que les créateurs placent sur le même plan agentivité et efficacité, détermination et réussite. La critique du néolibéralisme qui sous-tend les deux séries va main dans la main avec la reconnaissance du fait que la marge de manœuvre est bien ténue. Le droit au mouvement, je me suis efforcée de le montrer, s'entend ici non comme un droit hypothétique qu'il faudrait quémander, mais comme un droit effectif que l'on compte bien exercer. Qu'il doive être bafoué s'entend aussi, bien sûr. Les performances et photos des Kongo Astronauts et les objets chargés de Lamyne M expriment cette tension entre volonté et possibles. Les deux séries sont l'expression, simultanément, d'un être-en-tension - le vécu de ceux dont on entrave le mouvement - et d'une miseen-tension par ces mêmes acteurs, qui, à l'immobilité qu'on leur impose, opposent la force de leur être-au-monde.

Dominique MALAQUAIS

* Ce texte doit beaucoup à la générosité des artistes dont il est question: Eléonore Hellio et Michel Ekeba, fondateurs du collectif Kongo Astronauts, et Lamyne M. Je leur suis profondément reconnaissante. Je remercie vivement aussi Sarah Fila-Bakabadio, Barton Legum et Elisabeth Malaquais de leur lecture attentive de ces pages. Merci aussi à Axis Gallery (New York), qui a bien voulu partager avec moi images et informations concernant le travail de Lamyne M. Une version en anglais de cet article paraîtra à l'automne 2020 dans la revue américaine African Arts. 